\title{
Design, fabrication, and feasibility analysis of a colorimetric detection system with a smartphone for self- monitoring blood glucose
}

Hung-Chih Wang

Fuh-Yu Chang

Tung-Meng Tsai

Chieh-Hsiao Chen

Yen-Yu Chen 


\title{
Design, fabrication, and feasibility analysis of a colorimetric detection system with a smartphone for self-monitoring blood glucose
}

\author{
Hung-Chih Wang, ${ }^{a}$ Fuh-Yu Chang, ${ }^{a, \star}$ Tung-Meng Tsai, ${ }^{b}$ Chieh-Hsiao Chen, ${ }^{b, c}$ and Yen-Yu Chen \\ ${ }^{a}$ National Taiwan University of Science and Technology, Department of Mechanical Engineering Taipei, Taiwan \\ biXensor Co. Ltd., Taipei, Taiwan \\ ${ }^{\circ}$ China Medical University and Beigang Hospital, Taichung, Taiwan
}

\begin{abstract}
Maintaining appropriate insulin levels is very important for diabetes patients. Effective monitoring of blood glucose can aid in maintaining the body's insulin level, and thus reduce disease severities, secondary complications, and related mortalities. However, existing blood glucose measurement devices are inconvenient to carry and involve complex procedures, reducing the willingness of diabetes patients to regularly measure blood glucose. We aim to provide a rapid, convenient, and portable meter for diabetes patients. We introduce an integrated blood glucose detection device (IBGDD) that has no electronic component and uses the optical sensing module of a smartphone to inspect colorimetric blood strips. To demonstrate accuracy conformance of the developed device to the ISO 15197:2013 standard for blood glucose measurement, 20 diabetes mellitus patients used the IBGDD with smartphones to measure their blood glucose level. The measurement results revealed an accuracy of 100\%, completely satisfying the requirements of the ISO 15197:2013 standard. Overall, our specially designed IBGDD with a smartphone could achieve high accuracy and convenient usage for the measurement of blood glucose concentration. Furthermore, the device is highly portable and simple to operate. This contributes toward achieving self-monitoring of blood glucose by diabetes patients and improved mobile health in the future. ( ) The Authors. Published by SPIE under a Creative Commons Attribution 4.0 Unported License. Distribution or reproduction of this work in whole or in part requires full attribution of the original publication, including its DOI. [DOI: 10.1117/1.JBO.24.2 .027002]
\end{abstract}

Keywords: blood glucose; colorimetric blood strip; diabetes; smartphone.

Paper 180557R received Sep. 30, 2018; accepted for publication Jan. 22, 2019; published online Feb. 21, 2019.

\section{Introduction}

Rapid development of the economy and subsequent changes in lifestyle have led to the prevalence of diabetes, affecting over 382 million people in 2013. This number is expected to rise to 592 million by 2035 , which will impose a large and increasing global health burden. ${ }^{1}$ Some studies pointed out that frequent monitoring of blood glucose changes and effective control of blood glucose levels can help reduce the incidence of diabetic complications. ${ }^{2-8}$ However, current devices for blood glucose monitoring are inconvenient to carry or their testing procedures are complicated. Therefore, the willingness of patients to detect blood glucose frequently is reduced. This could seriously impact the health of diabetic patients with high blood glucose levels.

In general, glucose oxidase (GOx), glucose dehydrogenase $(\mathrm{GDH})$, and hexokinase are used in existing glucose measurement techniques, which are based on the principles of enzymatic reactions. ${ }^{9}$ Each enzyme has characteristic advantages and limitations, including redox potentials, cofactors, turnover rates, and selectivity for glucose. ${ }^{10}$ Glucose biosensors for selfmonitoring of blood glucose (SMBG) are usually based on the two enzyme families, GOx and GDH. GOx is the standard enzyme for biosensors. It is easy to obtain, cheap, can withstand greater extremes of $\mathrm{pH}$, ionic strength, and temperature, and

*Address all correspondence to Fuh-Yu Chang, E-mail: fychang @ mail.ntust .edu.tw has a relatively higher selectivity for glucose than many other enzymes. Thus, the manufacture of GOx involves less stringent conditions than other enzymes. ${ }^{10,11}$ The majority of current glucose biosensors are of the electrochemical type because of their higher sensitivity, reproducibility, and easy maintenance. Electrochemical sensors may be divided into potentiometric, amperometric, or conductometric types. ${ }^{12-14}$ However, electrochemical sensors have two disadvantages: one is interferences with nonspecific electroactive particles and the other is complications related to signal conversion. ${ }^{15,16}$

In the colorimetric method for SMBG, blood glucose test strips with special enzymes are used to produce chemical reactions with blood glucose, and changes in the glucose concentration are monitored by evaluating changes in the color of the strips. The conventional colorimetric method employs visual comparison to determine the value of blood glucose. Although this method has the advantages of low cost without an additional blood glucose meter, it has a serious drawback of low accuracy, especially due to differences in the personal visual evaluation. To overcome this issue, many researchers are currently attempting to apply image analysis software and hardware, such as a computer with a scanner or camera, to analyze the color value of blood glucose strips. ${ }^{17,18}$ These works provide a means to improve the accuracy of detection, but the significant and outstanding disadvantages lie in that they require additional and unportable hardware and complex operation with nonuserfriendly steps. To simplify the complex operation, smartphones have been used in several studies to detect the concentration 
of whole blood glucose. ${ }^{19-22}$ However, in these experiments, the measurement of the concentration of blood glucose was time consuming. Therefore, the development of a portable device with simple procedure is of high significance for regular SMBG.

In this study, a convenient and portable colorimetric SMBG system is introduced. The system combines a designed integrated blood glucose detection device (IBGDD) with an automatic glucose concentration analysis software installed in a smartphone. The IBGDD consists of a blood glucose test site (BGTS), a disposable lancet, a cover and baseplate set, and a light guide channel. The IBGDD is designed to have a minimum feature size and can be connected to the smartphone easily. In addition, the IBGDD does not have any electronic component and does not require any extra-light source during the detection of blood glucose concentration. Light from the smartphone's liquid crystal display (LCD) is reflected and guided to the detection area, as the required light source for capturing and analyzing color changes of blood glucose test strips. An optical simulation is performed to guide the light channel for achieving proper illuminance and uniform illumination on the BGTS. The smartphone's camera is used to capture the image of the strip, and then the software installed in the smartphone analyzes the image and provides accurate values of blood glucose concentration. For developing the automatic glucose concentration analysis software, whole blood samples with 10 different blood glucose concentrations, from 50 to $500 \mathrm{mg} / \mathrm{dL}$, were collected and a normalized algorithm and a line process were applied to establish reference mainlines for calculating the blood glucose concentration values. Finally, blood samples were collected from 20 diabetes mellitus patients and measured for blood glucose using the developed SMBG system with smartphone and a standard biochemical blood glucose analyzer. The measured results were compared and analyzed to confirm the accuracy and stability of the proposed SMBG system.

\section{Design and Simulation}

\subsection{IBGDD Design}

The major components of the IBGDD are the BGTS, a cover and baseplate set, a disposable lancet, and a light guide channel, as shown in Fig. 1. In our study, the smartphone's LCD was used as the light source to capture the BGTS image. In order to guide the light to the BGTS area efficiently, after the IBGDD 3-D model was built using the computer-aided design software, an optical simulation using Tracepro (Lambda Research Corporation) was performed to find the optimal design for the IBGDD, especially the reflector's angle.

\subsubsection{Blood glucose test site}

The main function of the BGTS is to observe changes in the color of test strip and measure the concentration of blood glucose. It is divided into two components: a test strip and a substrate. The substrate has a 2-mm hole, through which changes in the color of test strip after reaction can be observed. The diameter of the test strip is $2.5 \mathrm{~mm}$ and it is placed on the substrate with the same center as the through hole. To effectively observe and evaluate the color change in the observation area, optical simulation was performed in this study to determine the final design of the IBGDD with the optimal illuminance and illumination uniformity of the BGTS.

\subsubsection{Cover and baseplate set}

The main functions of the cover and baseplate are to lead the blood to the BGTS and block the ambient light source, which affects the image signal of BGTS. A black-colored blood guide hole made of hydrophilic acrylic plastic was designed on the cover. The hydrophilic property can effectively assist to lead the blood to the BGTS, and the black feature can completely isolate the ambient light.

\subsubsection{Light guide channel}

Because the direction of LCD light emission is parallel to the vertical direction of the BGTS, a light guide channel is required to redirect the light on the BGTS. According to the law of the light reflection theory, a reflect angle (RA) structure was designed in the guide light channel. This RA has an important effect on the brightness and illumination uniformity of the BGTS. The simulation experiment was aimed at determining an optimal RA of the light-guiding channel.

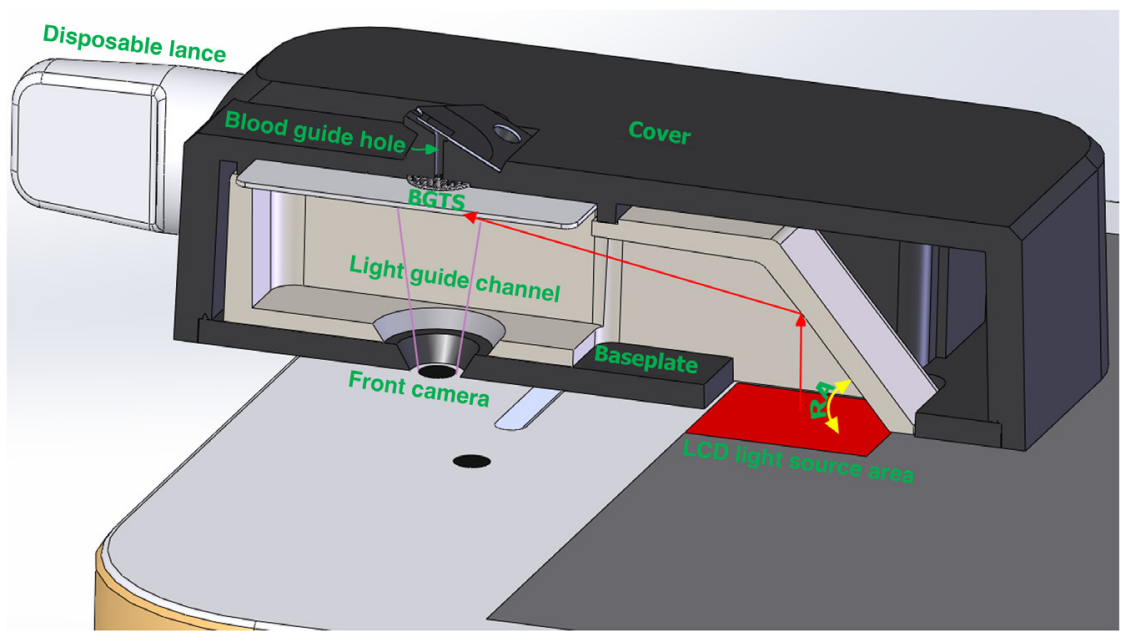

Fig. 1 Smartphone and IBGDD, comprising the BGTS, cover, baseplate, light guide channel, and disposable lancet. The red arrows indicate the light path in the IBGDD. 


\subsubsection{Disposable lancet}

The disposable lancet is mostly used to obtain blood from the fingers because it is currently the safest and easiest method. In this study, a special and small disposable lancet was designed such that it can work with our IBGDD and is convenient for users to carry. The lancet functions according to a spring mechanism, and the total size is only $6 \mathrm{~mm}$ in diameter and $37.9 \mathrm{~mm}$ in length. When the lancet is pushed against a finger, a hidden needle rapidly and gently pierces the finger. Then, the blood flows from the finger around the fingertip, and it is absorbed by the BGTS through capillary action when the finger touches the blood guide hole on the cover.

\subsection{Simulation and Measurement Method for Achieving Uniform Illumination of the BGTS Area}

The light source plays an important role in image analysis. To find an optimized RA that can guide light into the BGTS area, in this simulation experiment, the RA of the light guide channel was set from $30 \mathrm{deg}$ to $70 \mathrm{deg}$ with $5 \mathrm{deg}$ intervals as the analysis conditions. In this manner, the most optimal angle for the best illuminance and illumination uniformity on the observation area of the BGTS could be determined. The actual illumination of the smartphone's LCD was measured to be the light source illuminance value in the simulation. First, the screen was set to be white, and the screen brightness was raised to the maximum, and then a T10 illuminance meter (Konica Minolta, Japan) was used to measure the actual illumination of the screen. The average illumination is 230 lux. According to this measurement, the light source area was set to be $7.5 \mathrm{~mm} \times 6.3 \mathrm{~mm}$ in the simulation. The light wavelength was set by the general colorimetric method, in which the 550- nm wavelength is the most commonly used. The number of simulated traces was set to be 3 million.

The nine-point uniformity method was used to analyze the method of achieving uniform illumination in this study. The BGTS observation area was divided into nine symmetrical sampling areas, P1 to P9, as shown in Fig. 2. The sampling square size was $0.4 \mathrm{~mm} \times 0.4 \mathrm{~mm}$. The nine-area uniformity is defined by the minimum illuminance measured at the nine sampling areas divided by the maximum illuminance measured at the nine sampling squares, as

$$
U=\frac{\operatorname{Min}(P 1 \sim P 9)}{\operatorname{Max}(P 1 \sim P 9)},
$$

where $U$ is the BGTS uniformity.

\subsection{Simulation Result}

Our analysis of the simulation result indicates that the angle of reflection of the light guide channel has a significant effect on the illuminance and illumination uniformity of the BGTS observation area. At an angle of $30 \mathrm{deg}$, the illuminance and illumination uniformity are 50.43 lux and $90.43 \%$, respectively. When the angle increases, both illuminance and illumination uniformity continuously increase. At $50 \mathrm{deg}$, the illuminance and illumination uniformity reach the highest values of 53.67 lux and $95.47 \%$, respectively. With continued increase in the reflection angle, the illumination uniformity begins to decline. When the

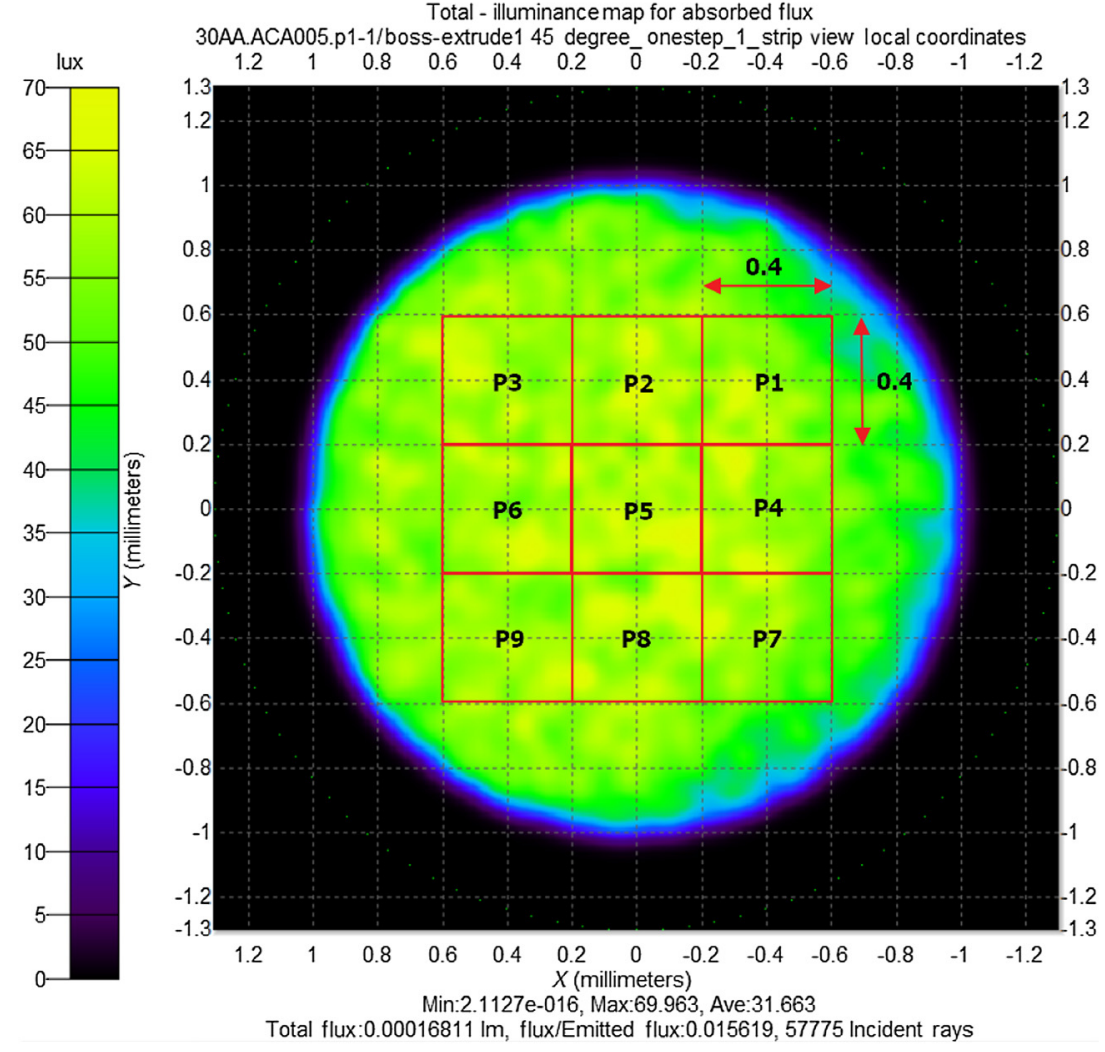

Fig. 2 Illuminance analysis of the BGTS area using the optical simulation program TracePro, with the BGTS area divided into nine symmetrical squares with sizes of $0.4 \mathrm{~mm} \times 0.4 \mathrm{~mm}$. 


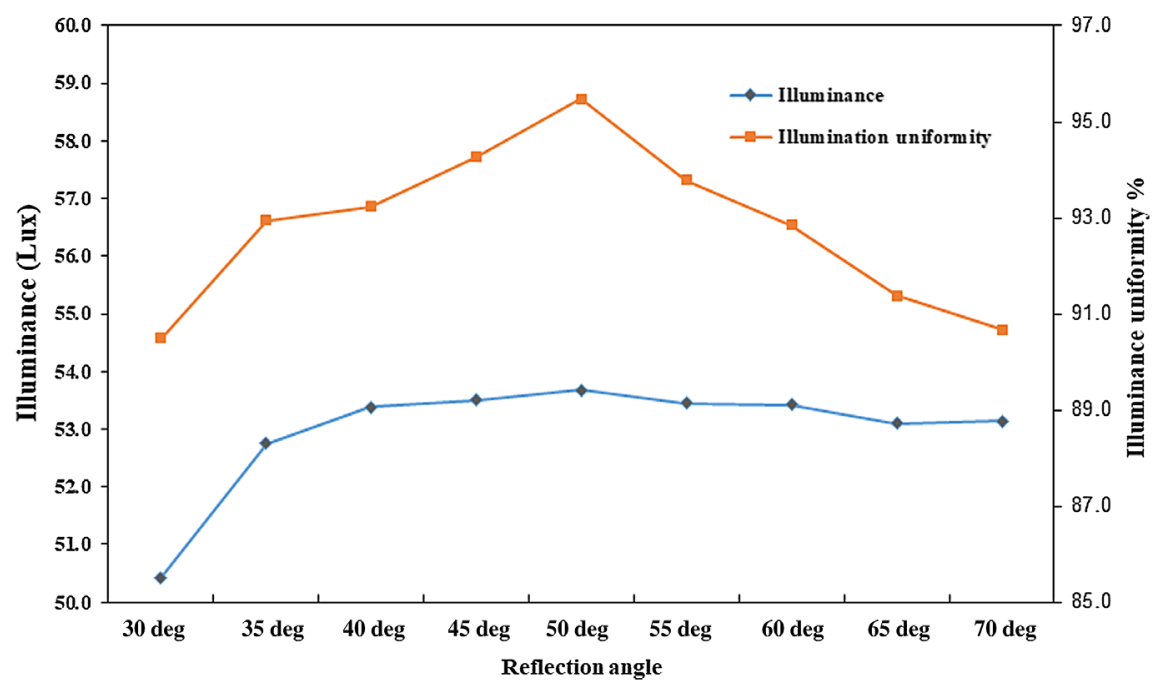

Fig. 3 Simulation results of illuminance and illuminance uniformity.

angle increases to $70 \mathrm{deg}$, the illumination uniformity declines to $90.68 \%$, as shown in Fig. 3. It is concluded that when the RA of the light guide channel is too low, it cannot effectively reflect the light to the BGTS, and therefore both illuminance and illumination uniformity are low. However, when the angle is higher than $50 \mathrm{deg}$, the light intensity gradually decreases toward the BGTS observation area, and therefore the illumination and illuminance uniformity of the observed area will decline. According to the simulation, the final design of RA of light guide channel was selected to be 50 deg to achieve the optimal illuminance and illumination uniformity.

\section{Experiment}

\subsection{Fabrication and Integration of the IBGDD}

All IBGDD components were fabricated by plastic injection molding, because the process allows components with consistent characteristics at reasonable production cost. After fabrication, all components were cleaned by ultrasonic cleaning and then assembled together. The mold of the reflector channel in the reflective area was polished, which can ensure a mirror-like surface roughness and enhance the light reflection efficiency of the reflector.

In this experiment, generally marketed products of colorimetric blood glucose strip were selected as the BGTS material, which had concentrations ranging from 40 to $500 \mathrm{mg} / \mathrm{dL}$. Since general strip dimension cannot meet IBGDD size a knife tool was used to cut the strip to the size $7.5 \mathrm{~mm} \times 6.3 \mathrm{~mm}$.

\subsection{Verification of the Illuminance and Illumination Uniformity of the BGTS Area}

The illuminance measuring instrument used is Konica Minolta T10 illuminance meter. In order to verify the basic illuminance and illumination uniformity, a standard card of $4 \mathrm{~mm}$ diameter was used. The standard card has Pantone White and completely uniform color characteristics. After inserting the standard card into the IBGDD, the glucose measurement program was started and the smartphone's LCD was switched on and guided to the BGTS area by the light guide channel, after which white balance locking and ISO value adjustment were performed. The program divided the BGTS area image into nine symmetrical sampling areas of square frame with four pixels each, and red, green, and blue (RGB) data from each rectangle image were collected to measure the minimum and maximum values. The measured data with the standard card were used to adjust the glucose measurement program for setting the basic illuminance and illumination uniformity. This operation was repeated five times to ensure the repeatability of the measurement.

\subsection{Preparation of Blood Glucose Samples with Different Concentrations}

In this experiment, a Yellow Springs Instrument (YSI) biochemical blood glucose analyzer equipment (YSI-2300) was used to establish the different concentrations of blood glucose from the collected whole blood samples.

Sterile heparin tubes were used to collect 120 c.c. venous blood samples from each patient at environmental temperature of $22^{\circ} \mathrm{C}$. After $24 \mathrm{~h}$ of standing, the blood glucose of the samples degraded to $0 \mathrm{mg} / \mathrm{dL}$, and then proper amounts of glucose solutions were added to the samples without blood glucose according to the required blood glucose concentration. After complete mixing with a rotor equipment, the YSI-2300 blood glucose meter was used to measure the blood glucose concentration.

\subsection{Colorimetric Enzyme Reaction Analysis and Establishing the Signal Reference Mainline}

The color of the colorimetric enzyme strip after reaction may vary with different blood glucose concentrations and reaction time. The first target of this experiment was to determine the signal of RGB specific and sensitive to the colorimetric enzyme strip and the time required to clearly distinguish the blood glucose concentration value of the samples. To avoid variations of blood glucose concentration caused by differences in temperature, the environmental temperature was set to $22^{\circ} \mathrm{C}$. After inserting the strip into the IBGDD, the glucose measurement program was started and the image of the BGTS area was captured by the smartphone's front camera (iPhone 5s, Apple Inc.) and the digital image of color change was separated into R, G, and B signals using the ColorAssist (FTLapps, Inc.) app according to the blood glucose concentration and reaction time. The concentration was adjusted from 50 to $500 \mathrm{mg} / \mathrm{dL}$ with an 
interval of $50 \mathrm{mg} / \mathrm{dL}$. The experiment was repeated three times for each of the 10 different blood samples. Subsequently, the normalized algorithm was applied to obtain 10 different concentration curves of RGB signals, which could assist in determining the signal most suitable for the colorimetric strip, and the determined signal was used to establish linear equations with the parametric regression analysis method. Normalization was performed by dividing the reaction signal value by the unreacted signal. Given that the normalized signal values for each concentration were too low so as to easily distinguish them, the range of the normalized signal values for each concentration was rescaled up to 400 times. Finally, the linear equations were used as the signal reference mainline to convert the imaged strip colors to the blood glucose concentration values.

\subsection{Verification Plan for the Developed SMBG System}

In 2013, ISO published a new standard ISO 15197:2013 and tightened the accuracy acceptance criteria. ${ }^{23}$ The standard stipulates that to satisfy the minimum acceptable accuracy for a glucose monitoring system, $95 \%$ of measurement results must fall within $\pm 15 \mathrm{mg} / \mathrm{dL}$ of the reference measured glucose concentration $<100 \mathrm{mg} / \mathrm{dL}$ or within $\pm 15 \%$ of the reference measured glucose concentration $\geq 100 \mathrm{mg} / \mathrm{dL}$. To verify the accuracy of the new SMBG system within the criteria of ISO 15197:2013, a verification test was performed with venous blood from 20 diabetic patients. The diabetic patients in this verification plan covered diabetes types 1 and 2 . The collected venous blood specimens were measured not only by the SMBG system but also by the YSI-2300 analyzer for accuracy comparison.

\section{Results and Discussion}

\subsection{Illuminance and Illuminance Uniformity of the BGTS Area}

The T10 illuminance meter was used to measure the illuminance of the BGTS area and the average illuminance was 54.6 lux from five times of measurement. The nine-point uniformity method was used to measure and analyze the RGB signals with the standard card in the BGTS area. The measured uniformity values for RGB signals from five times of measurement were all higher than $94.5 \%$. The values of $\mathrm{G}$ signal uniformity were significantly higher than those of the $\mathrm{R}$ and $\mathrm{B}$ signals, with an average value of $97.4 \%$. The coefficient of variation $(\mathrm{Cv})$ value of the RGB signals, ratio of the standard deviation to the mean value, was lower than $0.82 \%$. The $\mathrm{Cv}$ of the $\mathrm{G}$ signal reached as low as $0.27 \%$. This indicates that the designed reflector angle and reflection channel can provide uniform and stable light to the BGTS area. Uniformity and illuminance stability of the light source are extremely important factors affecting the accuracy and stability of the subsequent blood glucose measurement.

The measured illuminance and uniformity were also compared with the simulation results, and the comparison results proved the reliability of the simulation model. The simulation model will be used to improve the SMBG design in the future.

\subsection{Selection of the RGB Signal and Reference Mainline}

The developed SMBG system was applied to record the RGB signal values every $0.2 \mathrm{~s}$ for $50 \mathrm{~s}$, and each concentration sample was measured three times. The normalized algorithm was applied and 10 different concentration curves of RGB were obtained, as shown in Fig. 4. The figures show that the normalized curve of the $\mathrm{R}$ signal cannot be distinguished when the concentration is over $300 \mathrm{mg} / \mathrm{dL}$ [Fig. 4(a)], and the normalized curve of the $\mathrm{B}$ signal requires more than $30 \mathrm{~s}$ to distinguish each concentration curve [Fig. 4(b)]. In contrast, the 10 different concentration curves of the $\mathrm{G}$ signal could be distinguished completely after $10 \mathrm{~s}$, as shown in Fig. 4(b). Therefore, the normalized curves of the $\mathrm{G}$ signal were selected to establish the signal reference mainline, and normalized curve values of each concentration at $10 \mathrm{~s}$ were collected. Subsequently, the parametric regression analysis method was used to obtain a linear equation $y=-0.3247 x+219.25$, and the coefficient of determination $R^{2}$ was higher than 0.98 , as shown in Fig. 5. The linear equation was used as the reference mainline to convert the imaged strip colors to the blood glucose concentration.
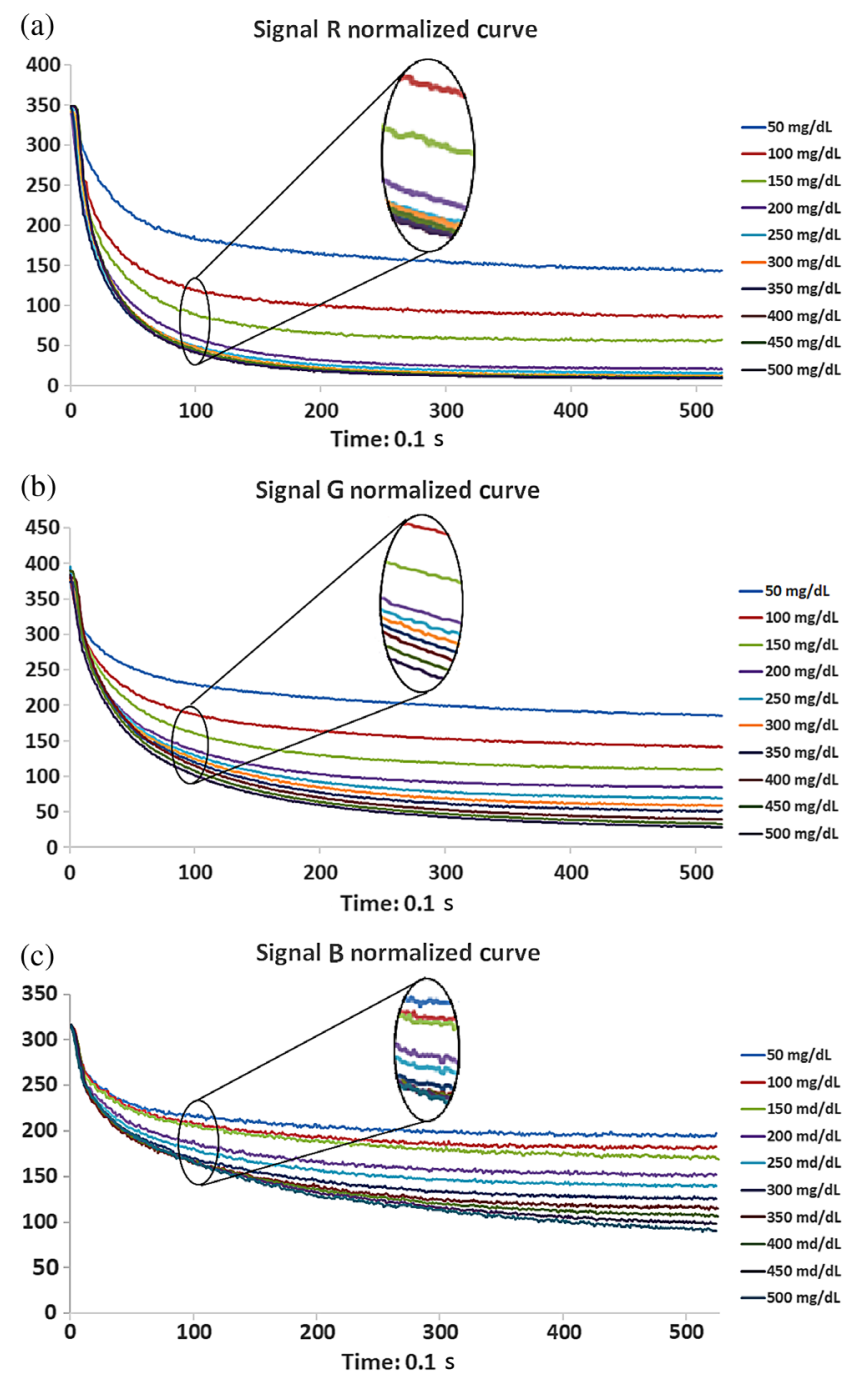

Fig. 4 Normalized curve from the measurement of 10 different concentration blood samples over $50 \mathrm{~s}$. Each curve represents the average of three measurements. (a) The $\mathrm{R}$ signal cannot be distinguished when the concentration reaches over $300 \mathrm{mg} / \mathrm{dL}$. (b) The 10 different concentration curves of the $\mathrm{G}$ signal can be clearly separated after $10 \mathrm{~s}$. (c) More than $30 \mathrm{~s}$ is required to distinguish the concentration curves of the B signal. 


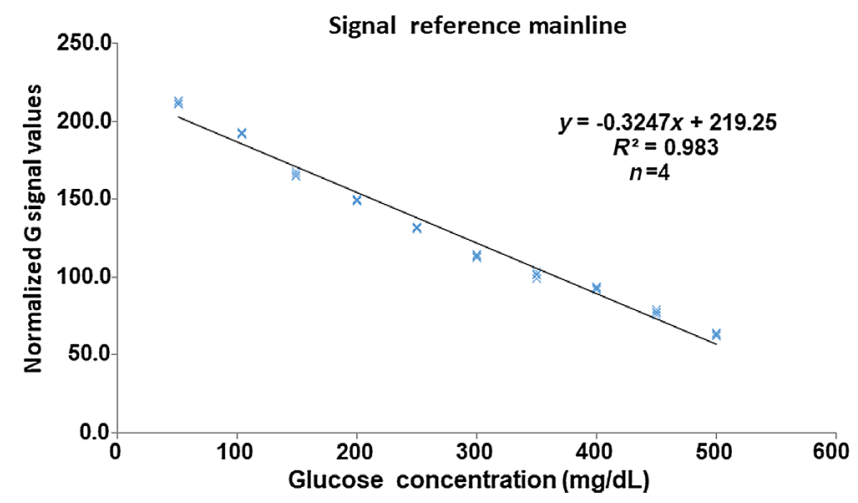

Fig. 5 Signal reference mainline obtained based on the normalized value of the $\mathrm{G}$ signal with the parametric regression analysis method. Glucose concentrations of $50 \sim 500 \mathrm{mg} / \mathrm{dL}$ were recorded with the IBGDD and $G$ signal and the results were read with a smartphone.

\subsection{Accuracy and Stability Tests for the Signal Reference Mainline}

To verify the accuracy and stability of the signal reference mainline, six different blood samples with glucose concentrations adjusted at 50,100, 200, 300, 400, and $500 \mathrm{mg} / \mathrm{dL}$ were tested and each sample was measured 10 times. The mean values of the samples from 10 times of measurement with the developed SMBG system were 49.0, 99.0, 198.3, 292.6, 397.0, and $488.7 \mathrm{md} / \mathrm{dL}$, respectively; their standard deviations were 1.2 , 2.9, 5.0, 9.6, 13.1, and 15.4, respectively; and their $\mathrm{Cv}$ values were $2.4 \%, 2.9 \%, 2.5 \%, 3.3 \%, 3.3 \%$, and $3.1 \%$, respectively. Compared with the adjusted glucose concentration of the samples, the accuracy of the measurements with the developed SMBG system was found to be $97.4 \%, 95.9 \%, 95.7 \%$, $98.3 \%, 97.4 \%$, and $95.8 \%$, respectively. As the experimental results show accuracy values over $95 \%$ for all tested samples and all $\mathrm{Cv}$ values under $3.8 \%$, the developed SMBG system with the established signal reference mainlines meet the standard criteria and clinical trial requirement for measuring blood glucose concentration with high accuracy and stability. The seven samples were also measured by the YSI-2300 analyzer for comparison. The concentration values were 50.4, 103.3, 207.3, 297.6, 407.8, and 510.2 mg/dL, respectively. Comparing these values, the developed system may measure the blood glucose concentration with the same level of accuracy and stability as the gold standard equipment.

\subsection{Verification Result for the Developed SMBG System}

In the verification with venous blood collected from 20 diabetes patients, 4 were diabetes type 1 patients and 16 were diabetes type 2 patients. The age distribution is as follows: $5 \%$ under the age of $20,15 \%$ between 21 and 30, 20\% between 31 and $40,25 \%$ between 51 and $60,10 \%$ between 61 and 70 , and $5 \%$ over the age of 71 . According to the analysis of data from the 20 patients, measurement results of the developed SMBG system and YSI-2300 analyzer were compared, and the data were found to completely satisfy the $\pm 15 \mathrm{mg} / \mathrm{dL}$ or $\pm 15 \%$ criteria, as shown in Fig. 5. The accuracy acceptance percentage is 100\% and meets the ISO 15197:2013 (E) criteria successfully. The parametric regression analysis of the data revealed a coefficient of determination $\left(R^{2}\right)$ value of 0.9848 , as shown in Fig. 6(b). These analyses show that the developed system measured the blood glucose concentration with the same level of accuracy and stability as the gold standard equipment. The design of the experiment and human subject involvement were approved by China Medical University Hospital.

\section{Conclusions}

In this study, a convenient and portable self-monitoring blood glucose system, comprising a special IBGDD and a smartphone, was developed and tested. As no additional light source and electronic part are required in the IBGDD, the device is as small as a pen cap or a key chain. An optical simulation was performed to optimize the design of the IBGDD in order to guide the light from the smartphone's LCD to the colorimetric strip efficiently. The smartphone's camera was used to capture the image of the colorimetric strip, and then the developed blood glucose concentration analysis program, installed in the smartphone, was used to obtain the measured value of blood glucose concentration. To confirm the accuracy and stability of the developed system, data from blood samples of 20 diabetes patients were used to measure their blood glucose and the results were compared with measured data from a commercial YSI-2300 blood glucose analyzer. The analysis results showed that all measured data were within the $\pm 15 \mathrm{mg} / \mathrm{dL}$ or $\pm 15 \%$
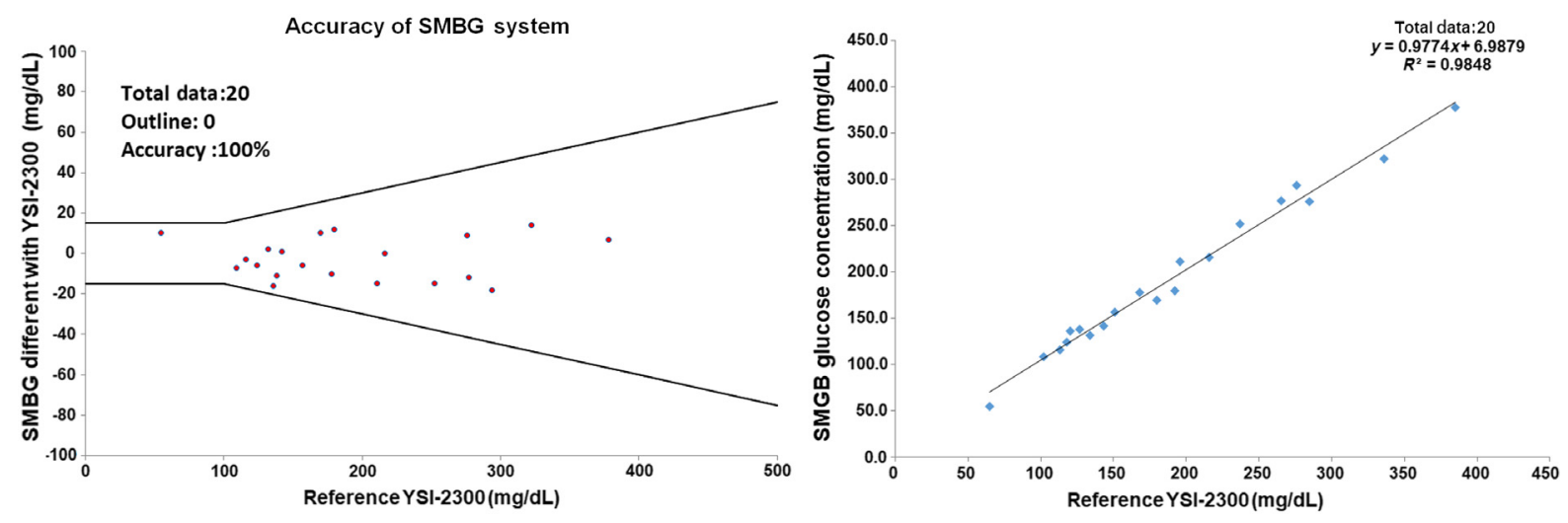

Fig. 6 ISO 15197:2013 and parametric regression analysis results. (a) Comparison of the measured data from the developed SMBG system and the YSI-2300 analyzer for 20 samples. The plot also gives the superimposed tolerance bands according to the ISO 15197:2013 criteria for accuracy. (b) Parametric regression analysis plot comparing results for the SMBG and YSI-2300 analyzer. 
criteria. The accuracy acceptance percentage was $100 \%$. The results prove that the developed system can completely meet the requirements of the ISO 15197:2013 standard for blood glucose measurement.

In the next phase plan, a clinical trial will be conducted with a hospital. It will enroll 120 subjects (type 1 and type 2 diabetes patients). These subjects will use the IBGDD with smartphones to measure their blood glucose levels. The measured data will be compared with those of the YSI-2300 analyzer to verify whether the clinical trial results meet the ISO 15197:2013 accuracy requirements.

In the future, our goal is to design a personal health management system that combines glycosylated hemoglobin (HbA1C), cholesterol, triglyceride, and blood glucose data. The personal health system data will be used to analyze physiological information, which will be sent to the cloud system of medical institutions via the internet. In this manner, concerned physicians can quickly grasp and judge the health information and transmit the related medical information to the patient. Through this personal health management system, patients can effectively employ digital technology to achieve physical health.

\section{Disclosures}

T. M. Tsai, C. H. Chen, and Y. Y. Chen are cofounders of iXensor Co., Ltd., and H. C. Wang was an employee of iXensor Co., Ltd. F. Y. Chang has no relevant conflict of interests to declare.

\section{Acknowledgments}

This research was supported by iXensor Co., Ltd., and approved by IRB of China Medical University Hospital (CMUH102REC2-050). Ministry of Science and Technology, Taiwan, Republic of China under Grant MOST 106-2221-E-011071-MY2.

\section{References}

1. L. Guariguata et al., "Global estimates of diabetes prevalence for 2013 and projections for 2035," Diabetes Res. Clin. Pract. 103(2), 137-149 (2014).

2. N. Poolsup, N. Suksomboon, and S. Rattanasookchit, "Meta-analysis of the benefits of self-monitoring of blood glucose on glycemic control in type 2 diabetes patients: an update," Diabetes Technol. Therapeut. 11(12), 775-784 (2009).

3. S. Skeie et al., "Self-monitoring of blood glucose in type 1 diabetes patients with insufficient metabolic control: focused self-monitoring of blood glucose intervention can lower glycated hemoglobin A1C," J. Diabetes Sci. Technol. 3(1), 83-88 (2009).

4. S. L. Tunis and M. E. Minshall, "Self-monitoring of blood glucose (SMBG) for type 2 diabetes patients treated with oral anti-diabetes drugs and with a recent history of monitoring: cost-effectiveness in the US," Curr. Med. Res. Opin. 26(1), 151-162 (2010).

5. E. I. Boutati and S. A. Raptis, "Self-monitoring of blood glucose as part of the integral care of type 2 diabetes," Diabetes Care 32(Suppl. 2), S205-S210 (2009).

6. L. Jovanovic, "Using meal-based self-monitoring of blood glucose as a tool to improve outcomes in pregnancy complicated by diabetes," Endocr. Pract. 14(2), 239-247 (2008).

7. M. J. O'Kane and J. Pickup, "Self-monitoring of blood glucose in diabetes: is it worth it?" Ann. Clin. Biochem. 46(4), 273-282 (2009).

8. R. Weiss and I. Lazar, "The need for continuous blood glucose monitoring in the intensive care unit," J. Diabetes Sci. Technol. 1(3), 412-414 (2007).

9. C. P. Price, "Point-of-care testing in diabetes mellitus," Clin. Chem. Lab. Med. 41(9), 1213-1219 (2003).
10. A. Heller and B. Feldman, "Electrochemical glucose sensors and their applications in diabetes management," Chem. Rev. 108(7), 2482-2505 (2008).

11. S. B. Bankar et al., "Glucose oxidase: an overview," Biotechnol. Adv. 27(4), 489-501 (2009).

12. K. Habermüller, M. Mosbach, and W. Schuhmann, "Electron-transfer mechanisms in amperometric biosensors," Fresenius' J. Anal. Chem. 366(6-7), 560-568 (2000).

13. J. Pearson, A. Gill, and P. Vadgama, "Analytical aspects of biosensors," Ann. Clin. Biochem. 37(2), 119-145 (2000).

14. D. R. Thevenot et al., "Electrochemical biosensors: recommended definitions and classification," Pure Appl. Chem. 71(12), 2333-2348 (1999).

15. S. Dzyadevych et al., "Amperometric enzyme biosensors: past, present and future," IRBM 29(2-3), 171-180 (2008).

16. B. H. Ginsberg, "Factors affecting blood glucose monitoring: sources of errors in measurement," J. Diabetes Sci. Technol. 3(4), 903-913 (2009).

17. D. J. Soldat, P. Barak, and B. J. Lepore, "Microscale colorimetric analysis using a desktop scanner and automated digital image analysis," J. Chem. Educ. 86(5), 617 (2009).

18. K. Tohda and M. Gratzl, "Micro-miniature autonomous optical sensor array for monitoring ions and metabolites 1: design, fabrication, and data analysis," Anal. Sci. 22(3), 383-388 (2006).

19. L. Shen, J. A. Hagen, and I. Papautsky, "Point-of-care colorimetric detection with a smartphone," Lab Chip 12(21), 4240-4243 (2012).

20. J. P. Devadhasan et al., "Whole blood glucose analysis based on smartphone camera module," J. Biomed. Opt. 20(11), 117001 (2015).

21. Y. Wang et al., "Smartphone spectrometer for colorimetric biosensing," Analyst 141(11), 3233-3238 (2016).

22. Y. Wu et al., "Clinical chemistry measurements with commercially available test slides on a smartphone platform: colorimetric determination of glucose and urea," Clin. Chim. Acta 448, 133-138 (2015).

23. International Organization for Standardization, "ISO 15197:2013. In vitro diagnostic test systems- Requirements for blood-glucose monitoring systems for self-testing in managing diabetes mellitus," (2013).

Hung-Chih Wang is a PhD candidate at the National Taiwan University Science and Technology, Taiwan. He received his BS and MS degrees in mechanical engineering from the National Taiwan University Science and Technology, Taiwan. He is experienced in mechanical product design, optical and biomedical device development for over 20 years.

Fuh-Yu Chang received his $\mathrm{PhD}$ in mechanical engineering, Leeds University, United Kingdom. Currently, he is working as an associate professor in the Mechanical Engineering Department of National Taiwan University Science and Technology, Taiwan. He has over 10 years of experience in biomedical device development. He is also interested in the semiconductor industry, electronics, and related optical design.

Tung-Meng Tsai received his $\mathrm{PhD}$ in chemistry engineering from the National Chung Hsing University, Taiwan. Currently, he is working in iXensor as chief executive officer and cofounder. He has more than 20 years of experience in biomedical device development.

Chieh-Hsiao Chen received his MD degree from KMU and $\mathrm{PhD}$ in biomedical engineering from NCKU, Taiwan. He specializes in nanotechnologies for cancer treatment, biosignal processing, and entrepreneurship. He works at iXensor as chief medical officer and cofounder. He also participates in clinical strategy, algorithms, and user experiences. He teaches biodesign and entrepreneurship at $\mathrm{CMU}, \mathrm{KMU}$, and NCKU. He is also the director of urology at $\mathrm{CMUH}$, Beigang, and CEO of Brain Navi Ltd.

Yen-Yu Chen received his $\mathrm{PhD}$ in electrical engineering from National Taiwan University, Taiwan. Afterward, he worked as a visiting researcher at Massachusetts Institute of Technology, California Institute of Technology, and Stanford University, to develop advanced optical imaging systems for biomedical research. He is now the CTO of iXensor and leads the company's technology and strategy development. His fields of interest include medical devices, optical instruments, and digital health. 